\title{
APPROACH TO EQUILIBRIUM FOR THE STOCHASTIC NLS
}

\author{
J. L. Lebowitz, Ph. Mounaix And W.-M. Wang
}

\begin{abstract}
We study the approach to equilibrium, described by a Gibbs measure, for a system on a $d$ dimensional torus evolving according to a stochastic nonlinear Schrödinger equation (SNLS) with a high frequency truncation. We prove exponential approach to the truncated Gibbs measure both for the focusing and defocusing cases when the dynamics is constrained via suitable boundary conditions to regions of the Fourier space where the Hamiltonian is convex. Our method is based on establishing a spectral gap for the non self-adjoint Fokker-Planck operator governing the time evolution of the measure, which is uniform in the frequency truncation $N$. The limit $N \rightarrow \infty$ is discussed.
\end{abstract}

\section{Contents}

1. Introduction

2. Truncated Fourier space formulation and the Theorem

3. Sobolev embedding and convex Hamiltonian

4. The Witten-Hodge complex and spectral gap

5. Exponential approach to equilibrium

6. The limit $N \rightarrow \infty$

7. Appendix: The Witten-Hodge complex 


\section{Introduction}

The nonlinear Schrödinger equation is used to model a variety of phenomena in different physical systems, see e.g., [LRS, MCL] and references therein. It comes in two versions: the focusing and the defocusing cases. These correspond respectively to the sign of $\lambda$ in front of the nonlinear term in the Hamiltonian $H$ of the (isolated) system,

$$
H:=H(u, \bar{u})=\int_{\mathbb{T}^{d}}\left(|\nabla u|^{2}+m|u|^{2}\right) d x+\frac{2 \lambda}{p} \int_{\mathbb{T}^{d}}|u|^{p} d x
$$

where $p$ is a positive even integer and $m \geq 0$. Here $u$ is a complex valued field (function) on the $d$-torus: $\mathbb{T}^{d}:=[0,2 \pi]^{d}$ after identifying the end points. The time evolution of $u$ satisfies the equation:

$$
\begin{aligned}
i \frac{\partial u}{\partial t} & =-\frac{\partial H}{\partial \bar{u}} \\
& =-\Delta u+m u+\lambda|u|^{p-2} u
\end{aligned}
$$

(Note that (1.1) differs from the $H$ in [LRS, MCL] by a factor of 2.)

In the defocusing case, $\lambda$ is positive, $H$ is bounded from below and one then has global existence and uniqueness of smooth solutions in $d \leq 4$ for appropriate $p$; more precisely, for $p \leq 4$ in $d=4, p \leq 6$ in $d=3$ and all $p$ in $d \leq 2$. This is the case of interest in constructive quantum field theory [GJ].

The situation is very different for the focusing case, $\lambda<0$. In this case $H$ is not bounded from below and there exists a critical $p_{c}, p_{c}=2+4 / d$, such that for $p<p_{c}$, there are unique global smooth solutions; for $p=p_{c}$ there are unique global smooth solutions with small $L^{2}$ norm. For $p>p_{c}$ but $p \leq 2 d /(d-2)$, one needs to have small $H^{1}$ data to have unique global solutions, cf. e.g., [Bo3].

This dichotomy carries over to the possible existence of a (non-truncated) Gibbs measure with density

$$
\mu=Z^{-1} \exp \left[-\beta \int_{\mathbb{T}^{d}} \frac{2 \lambda}{p}:|u(x)|^{p}: d x\right]
$$

with respect to the free field Gaussian measure, where $\beta>0$ and : : indicates Wick ordering, which is needed for $d \geq 2$ and $Z^{-1}$ is a normalization factor.

These measures exist for the defocusing case, $\lambda>0$ for all $p$ in $d=1$ (without Wick ordering) and for $p \leq 6$ in $d=2$, and $p \leq 4$ in $d=3[\mathrm{GJ}]$. Furthermore, despite the fact that this measure is supported on rough functions, Bourgain showed that it is invariant under the dynamics given by $(1.2)$ for $d \leq 2$ $[$ Bo1, 2]. This means that the dynamics can be defined (after Wick ordering modification in $d=2$ ) on a set of full measure with respect to $\mu$.

The focusing case is more delicate. Since $H$ is unbounded from below, it is obvious that the measure $\mu$ cannot exist without some restrictions on its domain. It was shown by Lebowitz, Rose and Speer in [LRS] that in $d=1$, the Gibbs measure exists for $p=4$ when restricted to $L^{2}$ balls and that it exists for $p=6$ with the additional condition of small $|\lambda|$, cf. also [McKV, Z]. In $d=2$, Jaffe [J] showed that the measure exists for $p=3$ for real $u$ when restricted to $L^{2}$ balls and after Wick ordering; while Brydges and Slade $[\mathrm{BS}]$ showed that this does not work when $p=4$.

In this paper, we shall deal with both the focusing and defocusing cases when the system is in contact with a heat reservoir of the Ornstein-Uhlenbeck type at reciprocal temperature $\beta$. This problem was studied in [MCL], whose formulation we shall follow here, cf. also [LRS]. The time evolution is then described by the stochastic nonlinear Langevin equation:

$$
d u+(\nu+i)\left(-\Delta+m+\lambda|u|^{p-2}\right) u d t=\sqrt{2 \beta^{-1} \nu} d W,
$$


where $\nu \geq 0$ is the friction and $W$ is a complex Wiener process, i.e., white noise in space and time. We note that for the moment, equation (1.4) is formal as it involves a very rough $L^{1}$ force on the right side.

To give a meaning to (1.4), we work in Fourier space and restrict the number of Fourier modes $n$ to be finite and constrain the Hamiltonian so that it remains bounded from below and is locally convex. We enforce this bound on the evolution by imposing appropriate Neumann boundary conditions in Fourier space. The problem then reduces to a finite dimensional Markov process on a compact space, whose stationary measure is given by a truncated Gibbs measure $\mu_{N}$. This is formulated in sect. 2 . We note that for fixed $N$, exponential approach to $\mu_{N}$ without any lower bound on the rate, follows from general probabilistic considerations, see [MCL].

The main new result of this paper is that the approach to $\mu_{N}$ is exponential with an exponent given by the size of the spectral gap for the non self-adjoint Fokker-Planck operator, which is uniform in the truncation $N$. This is proven in sects. 3-5. The proof is made possible by identifying the Fokker-Planck operator with a non self-adjoint Witten-Hodge Laplacian and a spectral analysis.

We mention that a related Witten-Hodge Laplacian in the self-adjoint setting was introduced by Sjöstrand [S1] to study correlation functions in equilibrium statistical mechanics and used by Wang [W] to study the parabolic Anderson model.

The question of what happens to the truncated measure $\mu_{N}$ and the dynamics in the limit as $N \rightarrow \infty$ is discussed briefly in sect. 6 . It appears that in $d=1$, the limit will coincide with the $\mu$ given in (1.3), whenever the latter exists. The situation in $d \geq 2$ is less clear since Wick ordering takes away convexity.

Acknowledgement. W.-M.W. thanks J. Sjöstrand for several useful conversations. J.L. and W.-M.W. thank D. Brydges and G. Gallavotti for clarifications on the construction of the Gibbs measure. J.L. and Ph.M. thank P. Collet for many helpful discussions. J.L. is partially supported by the grants NSF DMR 08021220 and AFOSR AF-FA9550-10; W.-M.W. is partially supported by the grant ANR-10-JCJC0109 and the Qiushi professorial chair of mathematics at Zhejiang University.

\section{Truncated Fourier space formulation and the Theorem}

Let $n \in \mathbb{Z}^{d}$ be the dual variable to $x \in \mathbb{T}^{d}$ and $a=\left\{a_{n}\right\}$ the dual of $u$ :

$$
u=\sum_{n \in \mathbb{Z}^{d}} e^{-i n \cdot x} a_{n}
$$

We rewrite equation (1.3) using Fourier series. Let $\Phi$ be the corresponding sum in the Fourier space for $H$ :

$$
\begin{aligned}
& \Phi(a) /(2 \pi)^{d}:=\Phi(a, \bar{a}) /(2 \pi)^{d} \\
& =\sum_{n \in \mathbb{Z}^{d}}\left(n^{2}+1\right)\left|a_{n}\right|^{2}+\frac{2 \lambda}{p} \sum_{j_{1}-j_{2} \ldots+j_{p-1}-j_{p}=0} a_{j_{1}} \bar{a}_{j_{2}} \ldots a_{j_{p-1}} \bar{a}_{j_{p}},
\end{aligned}
$$

where $a_{n} \in \mathbb{C}, n^{2}$ stands for $|n|^{2}$ and for simplicity we have set $m=1$. We now restrict to $u$ such that $a_{n}=0$ for $|n|>N$ and make estimates uniform in $N$, cf. the remark above (5.1) in sect. 5. Since all estimates are uniform, we will generally omit $N$ as a subscript, except in the statements of the Theorem and its Corollary. For notational simplicity, we set $\beta=2$ and rescale time by $(2 \pi)^{d}$.

We have the following equation for $a$ :

$$
\dot{a}_{n}=-i \frac{\partial \Phi}{\partial \bar{a}_{n}}-\nu \frac{\partial \Phi}{\partial \bar{a}_{n}}+\sqrt{\nu} \Gamma_{n}, \quad|n| \leq N,
$$


where the $\Gamma_{n}$ are independent centered complex Gaussian white noises with

$$
\left\langle\Gamma_{n}(t) \bar{\Gamma}_{n}\left(t^{\prime}\right)\right\rangle=2 \delta\left(t-t^{\prime}\right) .
$$

The Fokker - Planck equation describes the time evolution of the probability density $\tilde{P}(a, t)$ with initial distribution $\tilde{P}(a, 0)$ for a process given by a stochastic PDE. Conjugating by $e^{ \pm \Phi}$ and setting $P=e^{\Phi} \tilde{P}$, the Fokker - Planck equation for (2.2) has the form:

$$
\partial_{t} P(a, t)+L P(a, t)=0,
$$

where

$$
L=-\nu \sum_{n} \frac{\partial^{2}}{\partial \bar{a}_{n} \partial a_{n}}+\nu\|\nabla \Phi\|^{2}-\nu \Delta \Phi+\mathcal{H}_{\Phi}, \quad \nu>0 .
$$

Here

$$
\nabla \Phi \text { is the vector }\left\{\partial_{\bar{a}_{n}} \Phi, \partial_{a_{n}} \Phi\right\}
$$

$$
\Delta \Phi=\sum_{n} \frac{\partial^{2}}{\partial \bar{a}_{n} \partial a_{n}} \Phi
$$

and

$$
\mathcal{H}_{\Phi}=2 \sum_{n}\left(\partial_{a_{n}} \Phi \partial_{\bar{a}_{n}}-\partial_{\bar{a}_{n}} \Phi \partial_{a_{n}}\right)
$$

is the Hamiltonian vector field for the (finite dimensional) Hamiltonian $\Phi$ and is anti self-adjoint. (All indices above are restricted to $|n| \leq N)$. Thus the Fokker - Planck operator $L$ is non self-adjoint. This is a distinguishing feature of the present construction.

In order for the Gibbs measure $\mu_{N} \sim e^{-2 \Phi_{N}(a)}$ to be stationary for the Fokker-Planck equation (2.4) in the focusing case, we need to restrict to appropriate domains in $a$. Toward that end, we define the $H^{s}$ balls and the Hamiltonian $\mathbb{H}$-ball of radius $\sqrt{B}$ to be the set of $a$ such that, respectively

$$
\sum_{n \in \mathbb{Z}^{d}}\left(n^{2 s}+1\right)\left|a_{n}\right|^{2}<B, \quad B>0
$$

and

$$
|\Phi(a)| /(2 \pi)^{d}<B, \quad B>0,
$$

where $\Phi(a)$ is defined in (2.1).

Since we are considering $u$ such that $a_{n}=0$ for $|n|>N$, the sums in $(2.9,2.10)$ are restricted to $|n| \leq N$. We take as our domain the Hamiltonian $\mathbb{H}$-balls and impose adapted Neumann boundary conditions on $\partial \mathbb{H}$ - the energy surface:

$$
\left.\eta \cdot\left(-\nu \nabla \Phi-\nu \nabla+h_{\Phi}\right) P\right|_{\partial \mathbb{H}}=0,
$$

where $\eta$ is the normal direction, assuming that it is well-defined and

$$
h_{\Phi}=\left\{2 \partial_{a_{n}} \Phi,-2 \partial_{\bar{a}_{n}} \Phi\right\}
$$

is a vector, displayed using the same convention as in (2.6). On occasions, we will also call $h_{\Phi}$ the Hamiltonian vector field. It is important to remark that since $h_{\Phi}$ is tangential, the above boundary condition is equivalent to

$$
\left.\eta \cdot(-\nu \nabla \Phi-\nu \nabla) P\right|_{\partial \mathbb{H}}=0,
$$

which plays a pivotal role in establishing a spectral gap uniform in $N$.

In the focusing case, the $\mathbb{H}$-ball is not necessarily a connected set. The theorem below addresses the component that contains the origin. We call it the $\mathbb{H}_{0}$-ball. In the defocusing case, $\mathbb{H}_{0}$ coincides with $\mathbb{H}$.

Our main results are : 
Theorem. For $d=1,2, p \in 2 \mathbb{N}$ arbitrary, $d=3, p=4,6$ and $d=4, p=4$, there exist $C, c>0$ such that uniformly in $N$, the Fokker-Planck semi-group satisfies

$$
\left\|e^{-t L_{N}}-\Pi_{0}\right\|_{\mathcal{L}\left(L^{2}\left(\mathbb{H}_{0}\right), L^{2}\left(\mathbb{H}_{0}\right)\right)} \leq C e^{-c t}, \quad t \geq 0
$$

for sufficiently small $|\lambda|$, in a given Hamiltonian $\mathbb{H}_{0}$-balls with the Neumann boundary condition (2.11) on the Hamiltonian surface $\partial \mathbb{H}_{0}$, where $\Pi_{0}$ is the projection onto the unique ground state of $L_{N}$ :

$$
\psi_{N}=C_{N} e^{-\Phi_{N}}
$$

and $C_{N}$ is such that the truncated Gibbs measure $\mu_{N}:=\psi_{N}^{2}$ is normalized:

$$
\int_{\mathbb{H}_{0}} \psi_{N}^{2} \prod_{|n| \leq N} d a_{n} d \bar{a}_{n}=1,
$$

and we have indicated explicitly the truncation $N$ on the $\Phi($ a) defined in (2.1).

Corollary. In the defocusing case, the Theorem holds for all $\lambda \geq 0$ under the same conditions on $d$ and $p$.

We note that in the $1 \mathrm{~d}$ focusing case, for $p=4$ and 6 , the Theorem remains valid with the Hamiltonian $\mathbb{H}_{0}$-ball replaced by an $\mathbb{L}^{2}$-ball, cf. sect. 6. This is because the Hamiltonian vector field $h_{\Phi}$ is also tangential to the $\mathbb{L}^{2}$-sphere and (2.12) remains valid on the sphere. Without dissipation and forcing, this Hamiltonian geometry leads to the conservation of mass, i.e., the $L^{2}$ norm and energy, essential for the global existence and uniqueness of solutions for subcritical (or critical) dispersive NLS. With dissipation and forcing, the conservation laws are no longer there, but it is important to recognize that the geometry remains and is precisely what enables the present construction. The $1 \mathrm{~d}$ focusing case has particular interest as the limiting measure $\mu$ is known to exist [LRS], cf. sect. 6 .

Previous related results on stochastic NLS, e.g. [EH, KuS, O] pertain either to the focusing $L^{2}$ subcritical case, namely $p \leq 4$, the $1 \mathrm{~d}$ focusing case, where there are no blow-up solutions, or to the defocusing case. The forces there are smooth in the spatial variable $x$. So one works directly with the (non-truncated) SNLS. Generally speaking, this approach does not yield the explicit form of the invariant measure. For results in the purely dissipative case, cf. [DD].

Equation (1.3) balances dissipation with a rough force. The main novelty of the present construction is to work directly in the phase - space (the cotangent bundle) using the Fokker - Planck operator and impose boundary conditions there. This enables us to smooth the rough force by convexity of the Hamiltonian and treat the critical cases.

\section{Sobolev embedding and convex Hamiltonian}

We establish convexity of $\Phi$ uniformly in $N$ and prove

Proposition. Under the same conditions on $d$ and $p$ as in the Theorem, the Hessian of the truncated $\Phi$ satisfies

$$
c \mathbb{I}<\text { Hess } \Phi:=\Phi^{\prime \prime}<C \mathbb{I},
$$

in the $H^{1}$ ball defined in (2.9), where $c, C>0$ are constants which do not depend on $N$, if $|\lambda|$ is sufficiently small. Here Hess $\Phi$ is considered as an operator on $\ell^{2}\left([-N, N]^{d}\right) \times \ell^{2}\left([-N, N]^{d}\right)$. If $\Phi$ is defocusing, then $\lambda$ can be taken to be 1 and $C=C(B)$. 
Proof. We write the matrix operator $\Phi^{\prime \prime}$ in the form $\Phi^{\prime \prime}=M_{1}+M_{2}$, where

$$
\begin{aligned}
& M_{1}=\left(\begin{array}{cc}
{\left[\left[\partial_{\bar{a}_{j}} \partial_{a_{k}} \Phi\right]\right]} & 0 \\
0 & {\left[\left[\partial_{a_{j}} \partial_{\bar{a}_{k}} \Phi\right]\right]}
\end{array}\right), \\
& M_{2}=\left(\begin{array}{cc}
0 & {\left[\left[\partial_{\bar{a}_{j}} \partial_{\bar{a}_{k}} \Phi\right]\right]} \\
{\left[\left[\partial_{a_{j}} \partial_{a_{k}} \Phi\right]\right]} & 0
\end{array}\right),
\end{aligned}
$$

and [[ ] ] denotes the matrix of second order partial derivatives.

Now to prove (3.1), it suffices to prove that

$$
\left(v, \Phi^{\prime \prime} v\right) \geq c(v, v)
$$

for all $v=\left\{w_{j}, \bar{w}_{j}\right\} \in \ell^{2} \times \ell^{2}$. The quantity $\left(v, \Phi^{\prime \prime} v\right)$ is a sum of terms of the form $M_{1, j k} w_{j} \bar{w}_{k}$ or $M_{2, j k} w_{j} w_{k}$ and their complex conjugates. Let $W$ be the function with Fourier coefficients $w_{j}$ and $u$ the function with Fourier coefficients $a_{j}(|j| \leq N)$. We observe that since $\Phi$ is a sum of homogeneous polynomials in $a_{j}, \bar{a}_{k}$, the terms that occur in the sum are exactly the same as the terms in the evaluation of the following integrals:

$$
\begin{gathered}
\|W\|_{H^{1}}^{2}:=\|\nabla W\|_{L^{2}}^{2}+\|W\|_{L^{2}}^{2}=\sum\left(n^{2}+1\right)\left|w_{n}\right|^{2} \\
\left\|u^{(p / 2-1)} W\right\|_{L^{2}}^{2}, \int W^{2} \bar{u}^{2}|u|^{(p-4)} d x \quad \text { and its complex conjugate, }
\end{gathered}
$$

where here and below $\int \cdot d x$ denotes integration over the torus $\mathbb{T}^{d}$.

More precisely,

$$
\left(v, \Phi^{\prime \prime} v\right)=2\|W\|_{H^{1}}^{2}+\lambda p\left\|u^{(p / 2-1)} W\right\|_{L^{2}}^{2}+\lambda(p-2) \operatorname{Re} \int W^{2} \bar{u}^{2}|u|^{p-4} d x .
$$

Using now the inequalities

$$
\frac{2}{p}\left\|u^{(p / 2-1)} W\right\|_{L^{2}}^{2} \leq\left\|u^{(p / 2-1)} W\right\|_{L^{2}}^{2}+(1-2 / p) \operatorname{Re} \int W^{2} \bar{u}^{2}|u|^{p-4} d x \leq 2\left\|u^{(p / 2-1)} W\right\|_{L^{2}}^{2},
$$

we have in the focusing case,

$$
2\|W\|_{H^{1}}^{2}-2|\lambda| p\left\|u^{(p / 2-1)} W\right\|_{L^{2}}^{2} \leq\left(v, \Phi^{\prime \prime} v\right) \leq 2\|W\|_{H^{1}}^{2}+2|\lambda| p\left\|u^{(p / 2-1)} W\right\|_{L^{2}}^{2}
$$

and in the defocusing case

$$
2\|W\|_{H^{1}}^{2} \leq\left(v, \Phi^{\prime \prime} v\right) \leq 2\|W\|_{H^{1}}^{2}+2|\lambda| p\left\|u^{(p / 2-1)} W\right\|_{L^{2}}^{2} .
$$

Standard Sobolev embedding on $\mathbb{T}^{d}$, namely

$$
\|\cdot\|_{L^{p}} \leq C_{d, p}\|\cdot\|_{H^{d / 2(1-2 / p)}}
$$

then gives under the same conditions as in the Theorem on $d$ and $p$ that

$$
\left\|u^{(p / 2-1)} W\right\|_{L^{2}}^{2} \leq C_{d, p}\|u\|_{H^{1}}^{p-2}\|W\|_{H^{1}}^{2} .
$$

Using $(3.11)$ in $(3.8,3.9)$ and also smallness of $|\lambda|$ in $(3.8)$, proves the proposition. 


\section{The Witten-Hodge Laplacian and spectral gap}

We now prove that the Fokker-Planck operator $L$ in (2.5) restricted to Hamiltonian $\mathbb{H}$-balls defined in (2.9) has a spectral gap uniform in $N$ when the Hamiltonian is convex. We use the Witten-Hodge Laplacian formulation introduced in a related context in [S1]. We summarize some of the basic notions in the Appendix using the self-adjoint setting. Here we show that it corresponds to the operator $L$ and gives the desired spectral gap.

The non self-adjoint Fokker-Planck operator

Let $d$ be the exterior differentiation

$$
d:=\sum_{n} \partial_{b_{n}} d b_{n}^{\wedge}
$$

where $b_{n}$ stands for $a_{n}$ and $\bar{a}_{n}$ and

$$
d_{\Phi}:=e^{-\Phi} d e^{\Phi}=\sum_{n}\left(\partial_{b_{n}}+\partial_{b_{n}} \Phi\right) d b_{n}^{\wedge} .
$$

We note that here we work on $\mathbb{C}^{\Lambda}$ with $\Lambda=[-N, N]^{d}$, which could be identified with $\left(\mathbb{R}^{2}\right)^{\Lambda} \sim \mathbb{R}^{2|\Lambda|}$.

Let

$$
A=\left(\begin{array}{cc}
\nu \mathbb{I} & \mathbb{I} \\
-\mathbb{I} & \nu \mathbb{I}
\end{array}\right)
$$

where the notation is the same as in (3.2) with each bloc being one of the four possible sectors: $\bar{u} u, \bar{u} \bar{u}, u u, u \bar{u}$. Define the (formal) adjoint of $d_{\Phi}$ with respect to $A$ to be:

$$
d_{\Phi}^{*, A}=\sum_{n}\left(-\partial_{\bar{b}_{n}}+\partial_{\bar{b}_{n}} \Phi\right) \circ A d \bar{b}_{n}^{\rfloor} .
$$

(Properly speaking, $A$ is a map from the cotangent space $\left(\mathbb{C}^{[-N, N]^{d}}\right)^{*}$ to the tangent space $\mathbb{C}^{[-N, N]^{d}}$.) The Witten-Hodge Laplacian is then defined as

$$
-\Delta_{\Phi, A}=d_{\Phi}^{*, A} d_{\Phi}+d_{\Phi} d_{\Phi}^{*, A},
$$

cf. the paper of Bismut [Bi] for the general construction and also the Appendix in sect. 7 .

The reason that we introduce the Witten-Hodge Laplacian is that when restricting to scalar functions, the 0-forms, it is precisely the Fokker - Planck operator $L$ in (2.5) and we have the following identities:

$$
-\Delta_{\Phi, A}^{(0)}=d_{\Phi}^{*, A} d_{\Phi}=d_{\Phi}^{*} \circ A \circ d_{\Phi}=L,
$$

where

$$
d_{\Phi}^{*}=\sum_{n}\left(-\partial_{\bar{b}_{n}}+\partial_{\bar{b}_{n}} \Phi\right) d \bar{b}_{n}^{\rfloor} .
$$

We will also need it on 1-forms:

$$
-\Delta_{\Phi, A}^{(1)}=-\Delta_{\Phi, A}^{(0)} \otimes \mathbb{I}+2 \Phi^{\prime \prime} \circ A^{t},
$$

cf., [HHS, S2].

Remark. This is occasionally dubbed the supersymmetric approach, cf. e.g. [TT-NK] for the physics literature.

Let the Hamiltonian $\mathbb{H}$-ball be as defined in (2.10) and as before, $\mathbb{H}_{0^{-}}$-ball the connected component containing the origin. The following basic spectral characterization of the Fokker -Planck operator follows readily from (4.2). 
Lemma 1. Assume $\mathbb{H}_{0}$ is a convex set. The spectrum of $L$ in this ball with the Neumann boundary condition (2.11) is contained in the sector

$$
\{\kappa \in \mathbb{C}|\operatorname{Re} \kappa \geq \nu| \operatorname{Im} \kappa \mid\}
$$

and 0 is a simple eigenvalue.

Proof. The spectrum of $L, \sigma(L)$ is contained in the numerical range of $L$ :

$$
\{(u, L u) \in \mathbb{C} \mid u \text { in the domain of } L\} .
$$

From (4.2) for all $u$ in the domain of $L$ satisfying the Neumann boundary condition (2.11),

$$
(u, L u)=\left(u,-\Delta_{\Phi, A}^{(0)} u\right)=\nu \int_{\mathbb{H}_{0}}\left|d_{\Phi} u\right|^{2} d a d \bar{a}+i \operatorname{Im} \int_{\mathbb{H}_{0}}\left(d_{\Phi} u\right)^{2} d a d \bar{a},
$$

where $\left(d_{\Phi} u\right)^{2}$ denotes the sum of the square of components, with each component defined as in (4.1). Equation (4.5) implies (4.4), since (4.5) equals zero if and only if $d_{\Phi} u=0$, this means $u$ is a multiple of $e^{-\Phi}$, which concludes the proof.

Using the Witten-Hodge Laplacian (4.3) on 1-forms, we have, moreover, the following spectral gap lemma, essential for the proof of the Theorem.

Lemma 2. Assume that in the $\mathbb{H}_{0}$-ball, $\Phi^{\prime \prime}$ satisfies $c I<\Phi^{\prime \prime}<C I$ for some $c, C>0$. Then for some $c_{0}=c_{0}(c)>0$, the Fokker - Planck operator L with Neumann boundary conditions has the following properties uniformly in $N$ :

(i) $\{\sigma(L) \backslash\{0\}\} \cap\left\{z \in \mathbb{C} \mid \operatorname{Re} z<c_{0}\right\}=\emptyset$;

(ii) (up to constants) $e^{-\Phi}$ is the unique ground state with eigenvalue 0 .

Let $\Pi_{0}$ be the projection onto the normalized ground state. Then

(iii) $\|(L-z) u\| \geq\left(c_{0}-\operatorname{Re} z\right)\|u\|$ if $u=\left(1-\Pi_{0}\right) u$.

Proof. Assume $u$ is an eigenfunction of the Fokker -Planck operator $L$ with Neumann boundary conditions with eigenvalue $\kappa \neq 0$, i.e.,

$$
L u=-\Delta_{\Phi, A}^{(0)} u=\kappa u, \quad \kappa \neq 0 .
$$

Then from Lemma $1, d_{\Phi} u \neq 0$. Operating on equation (4.6) using $d_{\Phi}$ and taking the scalar product with $d_{\Phi} u$, we have

$$
\left(d_{\Phi} u,\left[d_{\Phi} d_{\Phi}^{*, A}\right] d_{\Phi} u\right)=\kappa\left(d_{\Phi} u, d_{\Phi} u\right) .
$$

Writing $v$ for $d_{\Phi} u$, we have equivalently

$$
\left(v,-\Delta_{\Phi, A}^{(1)} v\right)=\left(v,\left[-\Delta_{\Phi, A}^{(0)} \otimes \mathbb{I}+2 \Phi^{\prime \prime} \circ A^{t}\right] v\right)=\kappa(v, v),
$$

where we used (4.2).

Taking the real part of (4.6), we obtain

$$
\operatorname{Re} \kappa\|v\|^{2}=\left(v,\left[-\Delta_{\Phi}^{(0)} \otimes \mathbb{I}\right] v\right)+2 \nu\left(v, \Phi^{\prime \prime} v\right)
$$

where $-\Delta_{\Phi}^{(0)}=d_{\Phi}^{*} d_{\Phi}$ is the self-adjoint Laplacian on 0-forms. 
Using the convexity of $\Phi$, we then obtain (i) with $c_{0}=2 c \nu>0$ uniformly in $N$. Here we also used the fact that the self-adjoint Laplacian $-\Delta_{\Phi}^{(0)}$ has the same Neumann boundary condition as $-\Delta_{\Phi, A}^{(0)}$. We remark that in fact stronger results are known under appropriate conditions, namely

$$
\sigma\left(-\Delta_{\Phi, A}^{(0)}\right) \backslash\{0\} \subset \sigma\left(-\Delta_{\Phi, A}^{(1)}\right),
$$

cf. [HHS].

(ii) follows from (i) and Lemma 1. To prove (iii), we write

$$
\begin{aligned}
& \left.\|(L-z) u\|\|u\|=\left\|\left(-\Delta_{\Phi, A}^{(0)}-z\right) u\right\|\|u\| \geq \mid\left(-\Delta_{\Phi, A}^{(0)}-z\right) u, u\right) \mid \\
& \quad \geq\left|\operatorname{Re}\left(\left(-\Delta_{\Phi, A}^{(0)}-z\right) u, u\right)\right| \\
& \quad=\left|\left(\left(-\Delta_{\Phi}^{(0)}-\operatorname{Re} z\right) u, u\right)\right| \\
& \quad \geq\left(c_{0}-\operatorname{Re} z\right)(u, u)
\end{aligned}
$$

for all $u=\left(1-\Pi_{0}\right) u$.

Here we used self-adjointness of $-\Delta_{\Phi}^{(0)}$, the fact that up to constants $e^{-\Phi}$ is also the unique ground state of $-\Delta_{\Phi}^{(0)}$, i.e.,

$$
\Pi_{0}\left(-\Delta_{\Phi}^{(0)}\right)=\Pi_{0}\left(-\Delta_{\Phi, A}^{(0)}\right)
$$

and eigenfunction (of $-\Delta_{\Phi}^{(0)}$ ) expansion of $u$.

Remark. Since $L$ is non self-adjoint, it is no longer true that the resolvent at $z$ is bounded above by the inverse of the distance of $z$ to the spectrum. This is because of the non-commutativity of the self-adjoint and anti self-adjoint components and hence the necessity of the type of arguments in (4.8).

\section{Exponential approach to equilibrium}

Proof of the Theorem. We only need to verify the conditions in Lemmas 1 and 2, namely convexity of the Hamiltonian in $\mathbb{H}_{0}$. The rest will follow by applying these two lemmas and contour integration. Recall that the $H^{s}$ and the Hamiltonian $\mathbb{H}$-balls are as defined in $(2.9,2.10)$ and we restrict to functions $u$ such that its Fourier coefficients $a_{n}=0$ for $|n|>N$. So the sums below are to be understood as:

$$
\sum:=\sum_{n \in \mathbb{Z}^{d}}=\sum_{|n| \leq N}
$$

in other words, the $B$ below is fixed independent of $N$. Therefore the radius of the various "balls" are fixed. It is only the dimension of the balls that varies with $N$.

Remark. For most of the applications, $u=\pi \tilde{u}$, where $\pi$ is the projection onto the first " $N$ " Fourier modes while $\tilde{u}$ has full Fourier support and is in at least one of the "balls".

Define the Hamiltonian $\mathbb{H}$-ball as before:

$$
\begin{aligned}
\left|\Phi(a) /(2 \pi)^{d}\right|=\mid & \sum\left(n^{2}+1\right)\left|a_{n}\right|^{2}+\frac{2 \lambda}{p} \sum_{j_{1}-j_{2} \ldots+j_{p-1}-j_{p}=0} a_{j_{1}} \bar{a}_{j_{2}} \ldots a_{j_{p-1}} \bar{a}_{j_{p}} \mid \\
& <B .
\end{aligned}
$$

Using the results of sect. 3 , we have that if $a$ is in the above $\mathbb{H}$-ball, then for the defocusing case, $\lambda>0$, $\Phi$ is convex and $\left(v, \Phi^{\prime \prime} v\right)<C$. So $\mathbb{H}$ is a convex set with the well-defined boundary

$$
\partial \mathbb{H}=\left\{a \mid \Phi(a) /(2 \pi)^{d}=B\right\},
$$


and

for $a$ in $\mathbb{H}$.

$$
\sum\left(n^{2}+1\right)\left|a_{n}\right|^{2}<B
$$

For the focusing case, $\lambda<0$, assume we look at the connected component containing the origin, namely $\mathbb{H}_{0} \ni 0$. Let $A$ be the set

$$
A=\left\{\left.a\left|\sum\left(n^{2}+1\right)\right| a_{n}\right|^{2}<5 B\right\}
$$

and

$$
\mathbb{H}_{A}:=\mathbb{H}_{0} \cap A .
$$

The Proposition then gives that $\Phi$ is convex in $A$ and moreover $\Phi(\cdot)$ is equivalent to the $H^{1}$ norm:

$$
\frac{1}{2}\|f\|_{H_{1}}<\left(1-C|\lambda| B^{p}\right)\|f\|_{H_{1}}<\sqrt{\frac{\Phi(f)}{(2 \pi)^{d}}}<\left(1+C|\lambda| B^{p}\right)\|f\|_{H_{1}}<2\|f\|_{H_{1}},
$$

for $|\lambda| \ll 1$ and where $f$ is the function with Fourier coefficients $a \in A$.

Equation (5.3) together with (5.2) give that $\mathbb{H}_{0}$ is strictly contained in $A$ :

$$
\mathbb{H}_{A} \equiv \mathbb{H}_{0}
$$

So $\mathbb{H}_{0}$ is a convex set with the well-defined boundary

$$
\partial \mathbb{H}_{0}=\left\{a \mid \Phi(a) /(2 \pi)^{d}=B\right\} \subset A .
$$

We now evaluate the semi-group $e^{-t L}$ using the contour $\Gamma=\Gamma_{1}+\Gamma_{2}$, where $\Gamma_{1}$ is compact enclosing 0 and $\operatorname{Re} \Gamma_{1} \leq c^{\prime}<c_{0}$, the $c_{0}$ in Lemma 2 ; and $\Gamma_{2}$ is defined by

$$
\left\{\left.z|| \operatorname{Im} z|=| \operatorname{Re} z\right|^{2}+D \text {, if } \operatorname{Re} z>c^{\prime} \text { and }|\operatorname{Im} z| \leq c^{\prime 2}+D \text { if } \operatorname{Re} z=c^{\prime} \text { for some } D>0\right\} \text {, }
$$

so that

$$
\begin{aligned}
e^{-t L} & =\frac{1}{2 \pi i} \oint_{\Gamma} e^{-t z}(z-L)^{-1} d z \\
& =\frac{1}{2 \pi i} \oint_{\Gamma_{1}} e^{-t z}(z-L)^{-1} d z+\frac{1}{2 \pi i} \oint_{\Gamma_{2}} e^{-t z}(z-L)^{-1} d z .
\end{aligned}
$$

It follows from Lemmas 1 and 2 that the above contours lie in the resolvent set and the integrals are well-defined. (i, ii) of Lemma 2 proceed to give that the first term is $\Pi_{0}$ and (iii) gives the exponential estimate for the second term, uniformly in $N$ and concludes the proof.

Proof of Corollary. This follows from global convexity of $\Phi$, which is moreover uniform in the radius $\sqrt{B}$ of the $\mathbb{H}$-ball, since $H^{1}$ ball is equivalent to $\mathbb{H}$-ball in the defocusing case. Since

$$
d \Phi=\sum_{n} \frac{\partial \Phi}{\partial b_{n}} d b_{n}
$$

where $b_{n}=a_{n}$ and $\bar{a}_{n}$, let $u$ be the function with Fourier coefficients $\left\{a_{n}\right\}$, we have that

$$
\begin{aligned}
0<\sum\left|\frac{\partial \Phi}{\partial a_{n}}\right|^{2} & \leq 4 \pi^{2 d}\left(\|u\|_{H^{2}}+|\lambda|\|u\|_{L^{2 p-2}}^{p-1}\right)^{2} \\
& <4 \pi^{2 d}\left(\|u\|_{H^{2}}+|\lambda| C_{d, p}\|u\|_{H^{2}}^{p-1}\right)^{2}<\infty,
\end{aligned}
$$

where we used convexity for the lower bound, (3.10) and the restrictions on $d$ and $p$. So the energy surfaces $\partial \mathbb{H}$ is well-defined if $u \in H^{1} \cap H^{2}$. Using the density of $H^{2}$ in $H^{1}$, we reach the conclusion of the Corollary. 


\section{The limit $N \rightarrow \infty$}

As discussed in the introduction, the existence of a Gibbs measure $\mu \sim \exp [-\beta H]$ for the $H$ in $(2.1)$ is a problem which has been studied extensively. It corresponds in $d=1$ to the problem of the behavior of the limit $N \rightarrow \infty$ of the measure $\mu_{N} \sim \exp \left[-\beta \Phi_{N}\right]$. These measures exist for all $p$ in the defocusing case and for $p \leq 6$ for the focusing case under some restrictions. In $d \geq 2$, even the defocusing case requires Wick ordering, see (1.3), and it does not exist for the focusing case.

In $d=2$, the Hamiltonian dynamics corresponding to $\nu=0$, have been modified by Bourgain to include Wick ordering and shown, as mentioned earlier, to be well defined for the defocusing case when $p=4$. Whether one can make a similar modification to the Langevin dynamics, $\nu>0$, is an open and intriguing question. We note however that the Wick ordered nonlinear term $: u^{p}:$ is not convex. So the argument in this paper leading to the existence of a spectral gap would not hold for the modified dynamics. Of course the result might still be true. But this is another question.

The question of what happens to the dynamics generated by (2.2) or the Fokker - Planck equation (2.4) in the limit $N \rightarrow \infty$ is therefore of relevance here primarily in the case $d=1$. We consider the defocusing and the focusing cases below.

\section{The defocusing SNLS}

In this case the estimates in the Corollary are not only uniform in $N$, but also in the radius $\sqrt{B}$ of the Hamiltonian ball, which is equivalent to the $H^{1}$ norm. For $d=1$, letting $B \rightarrow \infty$ and subsequently $N \rightarrow \infty$, should therefore lead to the limiting measure with density:

$$
\mu=Z^{-1} \exp \left[-\frac{\beta \lambda}{p} \int_{\mathbb{T}}|u(x)|^{p} d x\right]
$$

with respect to the free field Gaussian measure. A full proof is under investigation.

\section{The $1 d$ focusing SNLS}

In the $1 d$ focusing case, the limiting measures when $p=4$ and for small $|\lambda|$ also $p=6$ are known to exist [LRS] and are invariant [Bo1] under the corresponding dispersive NLS dynamics, i.e., (1.3) when $\nu=0$. When $\nu>0$, the Theorem indicates a spectral gap uniform in $N$, albeit restricted to a Hamiltonian ball in which it is convex.

For fixed $N$, this restriction to a Hamiltonian ball can be replaced by a restriction to an $L^{2}$ ball with Neumann boundary conditions, as mentioned after the Theorem in sect. 2, since the Hamiltonian vector field is also tangential to the $L^{2}$ sphere. We then have that for both $p=4$ and 6 , the Hamiltonian is convex (uniformly in $N$ ) for small $|\lambda|$ depending on the radius of the $L^{2}$ ball using Sobolev embedding and interpolation. In the focusing case, the Hessian of the Hamiltonian is bounded above by a constant (which only depends on $N$ ). So using the same arguments as in sects. 4 and 5, the Fokker-Planck operator has a spectral gap uniform in $N$.

This $L^{2}$ Neumann restriction is a priori compatible with the ensuing $N \rightarrow \infty$ limit as the Brownian motion in $d=1$ is supported in $H^{1 / 2^{-}} \subset L^{2}$ and should lead to the limiting measure constructed in [LRS]. The question would then concern its invariance with respect to the SNLS dynamics in (1.3). The general procedure could be akin to that in [Bo1] on the corresponding Hamiltonian dynamics. Alternatively one could try to consider the limit $N \rightarrow \infty$ of the equation (2.4). See $[\mathrm{H}]$ for some results in a related but finite $N$ setting.

\section{Appendix: The Witten-Hodge complex}

Below we make a short introduction to the Witten-Hodge complex (originally introduced in [Wi]) using the self-adjoint setting. This is because the structure remains the same and the notations are a bit simpler, cf. Chap. 11, in particular Chap. 11.4 in [CFKS]. 
Toward that purpose, let $\phi \in C^{\infty}\left(\mathbb{R}^{N} ; \mathbb{R}\right)$. Let $d$ be the usual exterior differentiation:

$$
d=\sum_{j=1}^{N} \partial_{x_{j}} d x_{j}^{\wedge}
$$

and

$$
d_{\phi}=e^{-\phi} d e^{\phi}=d+d \phi^{\wedge}=\sum_{j \in \Lambda} z_{j} d x_{j}^{\wedge}
$$

where

$$
z_{j}=\frac{\partial}{\partial x_{j}}+\frac{\partial \phi}{\partial x_{j}} .
$$

(For the calculus of differential forms, see for example [Sp].)

If $f$ is a form of degree $m$, then $d_{\phi} f$ is a form of degree $m+1$. For example, if $f$ is a 0 -form, i.e., a scalar function in $C^{\infty}\left(\mathbb{R}^{N} ; \mathbb{R}\right)$, then

$$
d_{\phi} f=\sum_{j=1}^{N}\left(z_{j} f\right) d x_{j}
$$

is a 1-form, which we may identify with a vector valued function $F$ with the components:

$$
F_{j}(x)=\left(z_{j} f\right)(x),
$$

i.e., a function in $C^{\infty}\left(\mathbb{R}^{N} ; \mathbb{R}^{N}\right)$. (We note that when $\phi=0, d_{\phi} f=d f$, which is just the usual differential of $f$.) If $f$ is a 1 -form, $f=\sum_{j} f_{j} d x_{j}$ then

$$
d_{\phi} f=\sum_{i<j}\left(z_{i} f_{j}\right) d x_{i} \wedge d x_{j}
$$

is a 2-form, which we may identify with an $N \times N$ antisymmetric matrix function $M$ with the entries:

$$
M_{i j}(x)=-M_{j i}(x)=\left(z_{i} f_{j}\right)(x),
$$

i.e., a function in $C^{\infty}\left(\mathbb{R}^{N} ; \mathbb{R}^{N} \wedge \mathbb{R}^{N}\right)$. The operator $z_{j}$ can be seen as an annihilation operator in view of its action on $e^{-\phi}$, namely

$$
z_{j} e^{-\phi}=0, \text { for } j=1,2, \ldots, N \text {. }
$$

On the space of $m$-forms, one may define an $L^{2}$ scalar product for two $m$-forms $\omega$ and $v$ : $(\omega, v)$. Specializing to $v=d_{\phi} f$, where $f$ is an $(m-1)$ - form, we define the adjoint operator $d_{\phi}^{*}$ of $d_{\phi}$ so that:

$$
\left(\omega, d_{\phi} f\right)=\left(d_{\phi}^{*} \omega, f\right) .
$$

This gives the formal adjoint to be

$$
d_{\phi}^{*}=e^{\phi} d^{*} e^{-\phi}=\sum_{j=1}^{N} z_{j}^{*} d x_{j}^{\lrcorner},
$$

where

$$
z_{j}^{*}=-\frac{\partial}{\partial x_{j}}+\frac{\partial \phi}{\partial x_{j}}
$$

and $\rfloor$ is the usual contraction which lowers the degree of the forms. 
If $f$ is a form of degree $m$, then $d_{\phi}^{*} f$ is a form of degree $m-1$. For example, if

$$
f=\sum_{j=1}^{N} f_{j} d x_{j}
$$

is a 1 -form, then

$$
d_{\phi}^{*} f=\sum_{j=1}^{N} z_{j}^{*} f_{j}
$$

is a 0 -form, i.e., a scalar function in $C^{\infty}\left(\mathbb{R}^{N} ; \mathbb{R}\right)$. (We note that when $\phi=0, d_{\phi}^{*} f=d^{*} f$ is just the usual divergence of $f$.) If $f$ is a 0 -form, then $d_{\phi}^{*} f=0$.

The operator $z_{j}^{*}$ can be viewed as a creation operator. For example, when $N=1$ and $\phi=x^{2}$, $z^{*}:=z_{j}^{*}$ generates the first Hermite polynomial. We have the commutation relation:

$$
\left[z_{j}, z_{k}^{*}\right]=2 \partial_{j} \partial_{k} \phi
$$

which plays an important role. We check easily that indeed

$$
d_{\phi} d_{\phi}=d_{\phi}^{*} d_{\phi}^{*}=0 .
$$

Using $d_{\phi}, d_{\phi}^{*}$, we define the Witten Laplacian,

$$
-\Delta_{\phi}=d_{\phi}^{*} d_{\phi}+d_{\phi} d_{\phi}^{*}
$$

on $C^{\infty}\left(\mathbb{R}^{N} ; \wedge^{\ell} \mathbb{R}^{N}\right)(1 \leq \ell \leq N)$, where $\wedge^{\ell} \mathbb{R}^{N}$ denotes the $\ell^{\text {th }}$ anti-symmetric tensor product of $\mathbb{R}^{N}$ with $\wedge \mathbb{R}^{N}:=\mathbb{R}^{N}, \wedge^{2} \mathbb{R}^{N}:=\mathbb{R}^{N} \wedge \mathbb{R}^{N}$ etc. For example, if $\ell=2$, then an element of $C^{\infty}\left(\mathbb{R}^{N} ; \mathbb{R}^{N} \wedge \mathbb{R}^{N}\right)$ is an $N \times N$ anti-symmetric matrix valued function mentioned earlier and when $\ell=N$, it is the determinant.

Notice that

$$
d_{\phi} \Delta_{\phi}=\Delta_{\phi} d_{\phi}, d_{\phi}^{*} \Delta_{\phi}=\Delta_{\phi} d_{\phi}^{*}
$$

by using (7.6). If we let $\Delta_{\phi}^{(\ell)}$ be the restriction of $\Delta_{\phi}$ to forms of degree $\ell$, we obtain more precisely:

$$
d_{\phi} \Delta_{\phi}^{(\ell)}=\Delta_{\phi}^{(\ell+1)} d_{\phi}, d_{\phi}^{*} \Delta_{\phi}^{(\ell+1)}=\Delta_{\phi}^{(\ell)} d_{\phi}^{*} .
$$

We remark that the above construction is similar to that of Hodge Laplacian which corresponds to taking $\phi=0$. (For a quick overview of the analytical aspects of Hodge theory, see Chap 11.3 in [CFKS]. ) We have explicitly

$$
-\Delta_{\phi}^{(0)}=d_{\phi}^{*} d_{\phi}=\sum_{j=1}^{N} z_{j}^{*} z_{j}=-\sum_{j=1}^{N} \frac{\partial^{2}}{\partial x_{j}^{2}}+\|d \phi\|^{2}-\operatorname{Tr} \operatorname{Hess} \phi .
$$

For example, if $\phi$ is a non-degenerate quadratic form, then $-\Delta_{\phi}^{(0)}$ is an $N$-dimensional harmonic oscillator. $z_{j}$ and $z_{j}^{*}$ are just the annihilation and creation operators for the harmonic oscillator. More generally, we have

$$
\begin{aligned}
-\Delta_{\phi} & =\sum \sum z_{j} z_{k}^{*} d x_{j}^{\wedge} d x_{k}^{\lrcorner}+\sum \sum z_{k}^{*} z_{j} d x_{k}^{\lrcorner} d x_{j}^{\wedge} \\
& =\sum \sum z_{k}^{*} z_{j}\left(d x_{j}^{\wedge} d x_{k}^{\lrcorner}+d x_{k}^{\rfloor} d x_{j}^{\wedge}\right)+\left[z_{j}, z_{k}^{*}\right] d x_{j}^{\wedge} d x_{k}^{\lrcorner} \\
& =\sum z_{j}^{*} z_{j}+2 \sum \sum\left(\partial_{x_{j}} \partial_{x_{k}} \phi\right) d x_{j}^{\wedge} d x_{k}^{\rfloor} \\
& =-\Delta_{\phi}^{(0)} \otimes \mathbb{I}+2 \sum \sum_{13}\left(\partial_{x_{j}} \partial_{x_{k}} \phi\right) d x_{j}^{\wedge} d x_{k}^{\rfloor},
\end{aligned}
$$


where to obtain the third line from the second, we used (7.5). In particular, with the identification of 1 -forms with $\mathbb{R}^{N}$ valued functions, we obtain

$$
-\Delta_{\phi}^{(1)}=-\Delta_{\phi}^{(0)} \otimes \mathbb{I}+2 \phi^{\prime \prime}
$$

Since formally $\left(-\Delta_{\phi}^{(\ell)} u, u\right) \geq 0$, under appropriate conditions on $\phi$ at infinity, we can define $-\Delta_{\phi}^{(\ell)}$ as a self-adjoint operator and that $-\Delta_{\phi}^{(\ell)}$ has compact resolvent, cf. [S1]. Moreover $-\Delta_{\phi}^{(\ell)}$ has discrete spectrum contained in $[0, \infty)$. The lowest eigenvalue of $-\Delta_{\phi}^{(0)}$ is zero and a corresponding eigenfuction is $e^{-\phi}$, since this function is annihilated by $d_{\phi}$. This eigenvalue is simple, for if $u$ is another eigenfunction associated to the same eigenvalue, then $0=\left(-\Delta_{\phi}^{(0)} u, u\right)=\left\|d_{\phi} u\right\|^{2}$ and hence $d_{\phi} u=0$, which means precisely that $u$ is a multiple of $e^{-\phi}$.

Using (7.8), we obtain the following intertwining property of the spectra:

$$
\sigma\left(-\Delta_{\phi}^{(0)}\right) \backslash\{0\} \subset \sigma\left(-\Delta_{\phi}^{(1)}\right) .
$$

This is because if $f$ is an eigenfunction of $-\Delta_{\phi}^{(0)}$ :

$$
-\Delta_{\phi}^{(0)} f=\kappa f
$$

with $\kappa>0$, then operating on (7.14) with $d_{\phi}$, we obtain

$$
-d_{\phi} \Delta_{\phi}^{(0)} f=-\left(d_{\phi} d_{\phi}^{*}\right) d_{\phi} f=-\Delta_{\phi}^{(1)}\left(d_{\phi} f\right)=\kappa d_{\phi} f .
$$

So if $\kappa \neq 0$, then $d_{\phi} f \neq 0$ is an eigenform for $-\Delta_{\phi}^{(1)}$, which is the statement in (7.13).

This is in fact the main reason that we introduced $-\Delta_{\phi}^{(1)}$. Using $(7.12)$, we then obtain that $\sigma\left(-\Delta_{\phi}^{(0)}\right)$ has a spectral gap if $\phi$ is strictly convex. We end this self-adjoint introduction by remarking that if $e^{-\phi}$ is the eigenfunction of the Schrödinger operator

$$
-\sum \frac{\partial^{2}}{\partial x_{j}^{2}}+V
$$

for the lowest eigenvalue $\kappa$, then

$$
-\sum \frac{\partial^{2}}{\partial x_{j}^{2}}+V-\kappa=-\Delta_{\phi}^{(0)}
$$

From this point of view, the Witten-Hodge Laplacian can be seen as a natural generalization of harmonic oscillators when $\phi$ is quadratic.

\section{REFERENCES}

[Bi] J.-M. Bismut, The hypoelliptic Laplacian on the cotangent bundle, J. Amer. Math. Soc. 18 (2005), 379-476.

[Bo1] J. Bourgain, Periodic nonlinear Schrödinger equation and invariant measure, Commun. Math. Phys. 166 (1994), 1-26.

[Bo2] J. Bourgain, Invariant measure for the 2D-defocusing nonlinear Schrödinger equation, Commun. Math. Phys. 176 (1996), 421-445.

[Bo3] J. Bourgain, Nonlinear Schrödinger equations, IAS/Park City Mathematics Series (1999), 1-157. 
[BS] D. Brydges, G. Slade, Statistical mechanics of the 2-dimensional focusing nonlinear Schrödinger equation, Commun. Math. Phys. 182 (1996), 485-504.

[CFKS] H. L. Cycon, R. G. Froese, W. Kirsch, B. Simon, Schrödinger Operators, Springer-Verlag, 1987.

[DD] G. Da Prato, A. Debussche, Strong solutions to the stochastic quantization equations, Ann. of Prob. 31 (2003), 1900-1916.

[EH] J.-P. Eckmann, M. Hairer, Uniqueness of the invariant measure for a stochastic PDE driven by degenerate noise, Commun. Math. Phys. 219 (2001), 523-565.

[GJ] J. Glimm, A. Jaffe, Quantum Physics, Springer-Verlag, 1987.

[H] F. Herau, Short and long time behavior of the Fokker-Planck equation in a confining potential and applications, J. Func. Anal. 244 (2007), 95-118.

[HHS] F. Herau, M. Hitrik, J. Sjöstrand, Tunnel effect for Kramer-Fokker-Planck type operators, Ann Henri Poincaré 9 (2008), 209-274.

[J] A. Jaffe, Ann Arbor Lecture (1994).

[KuS] S. Kuksin, A, Shirikyan, Stochastic dissipative PDE's and Gibbs measure, Commun. Math. Phys. 213 (2000), 291-330.

[LRS] J. Lebowitz, R. Rose, E. Speer, Statistical mechanics of the nonlinear Schrödinger equation, J. Stat. Phys. 50 (1988), 657-687.

[McKV] H. McKean, K. Vaninski, Statistical mechanics of nonlinear wave equations. IV. Cubic Schrödinger, Comm. Math. Phys. 168 (1995), 479-491.

[MCL] Ph. Mounaix, P. Collet, J. Lebowitz, Nonequilibrium stationary state of a truncated stochastic nonlinear Schrödinger equation: Formulation and mean-field approximation, Phys. Rev. E 81 (2010).

[O] C. Odasso, Ergodicity for the stochastic complex Ginzburg-Landau equations, Annales de IHP, Probabilité et Statistique 42 (2006), 417-454.

[S1] J. Sjöstrand, Correlation asymptotics and Witten Laplacians, Algebra and Analysis 8 (1) (1996), 160-191.

[S2] J. Sjöstrand, Some results on non self-adjoint operators: a survey, Further progress in analysis, World Sci. Publ., Hackensack, NJ, 2009, pp. 45-75.

[Sp] M. Spivak, A Comprehensive Introduction to Differential Geometry, Vol. I, Publish or Perish, Berkely, 1970.

[TT-NK] J. Tailleur, S. Tanase-Nicola, J. Kurchan, Kramers equation and supersymmetry, J. Stat. Phys. 122(4) (2006), 557-595.

[W] W.-M. Wang, Supersymmetry, Witten complex and asymptotics for directional Lyapunov exponents in $\mathbb{Z}^{d}$, Ann. Henri Poincare 2 (2001), No. 2, 237-307.

[Wi] E. Witten, Supersymmetry and Morse theory, J. Diff. Geom. 17 (1982), 661-692.

[Z] P. Zhidkov, An invariant measure for the nonlinear Schrödinger equation, Dokl. Akad. Nauk SSSR $\mathbf{3 1 7}$ (1991), No. 3, 543-546.

Departments of Mathematics and Physics, Rutgers University, Piscataway, NJ 08854-8019,

E-mail address: lebowitz@math.rutgers.edu

Centre de Physique Théorique, UMR 7644 CNRS, Ecole Polytechnique, 91128 Palaiseau Cedex

E-mail address: philippe.mounaix@cpht.polytechnique.fr

CNRS and Department of Mathematics, Université Cergy-Pontoise, 95302 Cergy-Pontoise Cedex

E-mail address: wei-min.wang@math.cnrs.fr 